\title{
Ground State and Magnetostatic Properties of a Dipole System Arranged in Two-Dimensional Lattice
}

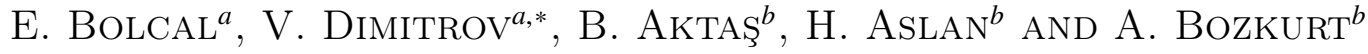 \\ ${ }^{a}$ Department of Physics, Istanbul Kultur University, Atakoy Campus E5 Karayolu Uzeri \\ Bakirkoy 34156 Istanbul, Turkey \\ ${ }^{b}$ Department of Physics, Gebze Institute of High Technology, Gebze, Turkey
}

\begin{abstract}
A system of nano sized modomain ferromagnetic particle arranged in two-dimensional lattice is theoretically investigated. The basic aim of the investigation is to find the ground state of these types of models where the interaction is long-ranged and anisotropic. The numerical calculations show that for some lattices there is a number of degenerated states near the zero temperature. The ground state of these systems is basically of a complicated antiferromagnetic type.
\end{abstract}

PACS: 75.10.-b

\section{Introduction}

There has been recently renewed interest in the magnetic properties of nanocomposite materials that consist of very fine magnetic particles embedded in a metallic or dielectric nonmagnetic host [1-7] and in nanocomposite materials that consist of multilayers with very thin magnetic layers $[8,9]$ which exhibit certain superparamagnetic (SPM) properties, too. If the dimension of a magnetic particle is reduced drastically, to a few nanometers, the domains in the particle merge into a single one, creating what is known as a single-domain particle and the total magnetic moment is the sum of all moments of the atomic particle. Therefore, the total magnetic moment of a single-domain nanoparticle can be about 10,000 times greater than the atomic moments of the constituent atoms. The behavior of such single-domain nanoparticles can be very complex due to the fact that the magnetic moment of each particle disturbs the behavior of neighboring particles. In general, this interaction between particles serves to align the magnetic moments in order to also reduce the energy of the whole. It is commonly believed that the magnetic behavior of a magnetic nanocomposite material is of SPM type. The complexity of the magnetic properties of nanocomposite magnetic materials is basically due to the dual nature of magnetic dipole-dipole interactions: if the dipoles are along a line their magnetic moment orientation is along the line (ferromagnetic ordering), however, when two dipoles are parallel then their magnetic moments are antiparallel (antiferromagnetic ordering).

The situation is much more complicated in a three-dimensional ensemble of magnetic dipoles. According to Hansen and Morup [1], in an assembly of particle magnetic moments at a given ordering temperature there is a transition from a superparamagnetic state to an ordered

\footnotetext{
* corresponding author; e-mail: recep.dimitrov@gmail.com
}

state resembling the spin-glass (SG) phase. However, according to Dorman et al. [5] no ordered state has yet been proved to exist in disordered nanoparticle assemblies, although there are some experimental clues in favor of its possible occurrence for strongly interacting particles. Some observed properties present analogies with those of spin glasses and it is reasonable to conjecture a low-temperature state similar to the SG phase, since the SG ingredients, i.e. disorder and frustration, are also present due to the disordered arrangement of particles, random distribution of easy axes and volume distribution, and the intrinsic conflicting character of dipoledipole interactions, which can be both ferromagnetic and antiferromagnetic. Thus, it seems that the creating of a rigorous theory of an ensemble of magnetic dipole system is an extremely difficult problem therefore further Monte Carlo (MC) studies are needed.

In order to better understand the behavior of a magnetic dipole ensemble "frozen in" a nanocomposite material, in the present study we mostly investigate the ground state configuration (at zero temperature) of such an ensemble. Since MC simulations at low temperatures are very slow and time consuming, in the present study we developed a special static relaxation (SR) method which finds very efficiently the minimum energy configuration (the ground state) of the dipole ensemble, and using the MC method warms up the system to higher temperatures.

\section{The model}

We consider a system of magnetic particles which are distributed on $N$ sites of a simple rectangle lattice with lattice parameters $\Delta x=\Delta y=a$. The particles are considered to be hard spheres, each carrying a central point dipole with a magnetic moment $\boldsymbol{m}$. The system is subject to a magnetic field which can be applied in any direction. The interaction energy between two point dipoles occupying lattice sites $i$ and $j$, is written as 


$$
\varepsilon_{l j}=-\frac{\mu_{0}}{4 \pi r_{i j}^{3}}\left[3\left(\boldsymbol{m}_{j} \cdot \boldsymbol{e}_{i j}\right)\left(\boldsymbol{m}_{j} \cdot \boldsymbol{e}_{i j}\right)-\left(\boldsymbol{m}_{j} \cdot \boldsymbol{m}_{i}\right)\right] .
$$

Thus, the Hamiltonian of the system is

$$
\begin{aligned}
H & =-\frac{1}{2} \sum_{i, j=1 ; j \neq i}^{N} \frac{\mu_{0}}{4 \pi r_{i j}^{3}}\left[3\left(\boldsymbol{m}_{j} \cdot \boldsymbol{e}_{i j}\right)\left(\boldsymbol{m}_{j} \cdot \boldsymbol{e}_{i j}\right)\right. \\
& \left.-\left(\boldsymbol{m}_{j} \cdot \boldsymbol{m}_{i}\right)\right]-\boldsymbol{B} \sum_{i=1}^{N} \boldsymbol{m}_{i} .
\end{aligned}
$$

Here, $\boldsymbol{m}_{i}, \boldsymbol{r}_{i}, \mu_{0}, \boldsymbol{B}$ is the dipole moment, the position vector of the $i$-th lattice site and permeability of free space, respectively; $r_{i j}=\left|\boldsymbol{r}_{j}-\boldsymbol{r}_{i}\right|, \boldsymbol{e}_{i j}=\frac{\boldsymbol{r}_{j}-\boldsymbol{r}_{i}}{r_{i j}}$. It is convenient to present Eq. (2) in a dimensionless form: if we assume $\boldsymbol{m}_{i}=n \mu_{\mathrm{B}} \boldsymbol{e}_{i}$ and $r_{i j}=r_{0} R_{i j}$ with $r_{0}$ the nearest neighbor distance, we get $H=E_{0} h$ where

$$
\begin{aligned}
& h=-\frac{1}{2} \sum_{i, j=1 ; j \neq i}^{N} \frac{1}{R_{i j}^{3}}\left[3\left(\boldsymbol{e}_{j} \cdot \boldsymbol{e}_{i j}\right)\left(\boldsymbol{e}_{j} \cdot \boldsymbol{e}_{i j}\right)\right. \\
& \left.\quad-\left(\boldsymbol{e}_{j} \cdot \boldsymbol{e}_{i}\right)\right]-\boldsymbol{b} \sum_{i=1}^{N} \boldsymbol{e}_{i}, \\
& E_{0}=\frac{\mu_{0} \mu_{\mathrm{B}}^{2}}{4 \pi r_{0}^{3}} n^{2}, \quad b=\frac{B \mu_{\mathrm{B}}}{E_{0}} .
\end{aligned}
$$

$E_{0}$ is the energy unit, $n$ is an integer number and $\mu_{\mathrm{B}}$ is the Bohr magneton. The first term in the above Hamiltonian represents the sum of all pairs of dipole-dipole interaction energies. The second term is the energy of the aligned dipoles in the direction of the applied magnetic field.

Considering the case of parallel moments for simplicity, the dipolar energy of a pair can be rewritten as

$$
\varepsilon_{i j}=-\frac{1}{R_{i j}^{3}}\left(3 \cos ^{2} \theta-1\right) .
$$

Expression (5) shows immediately the intricate character of the dipolar interaction as being: (a) long ranged because for a 3D assembly of dipoles one has to sum up over all pair energies and this sum is not absolutely convergent — the integral $\int \frac{r^{2} \mathrm{~d} r}{r^{3}}$ does not converge $\left(n(r) \square r^{2} \mathrm{~d} r\right.$ is the number of moment pairs separated by the distance $r$ ); (b) strongly anisotropic - leading to ferromagnetic coupling for $\cos 2(\theta)>1 / 3\left(\theta<54.75^{\circ}\right)$ or antiferromagnetic coupling for $\cos 2(\theta)<1 / 3\left(\theta>54.75^{\circ}\right)$.

These properties make it usually impossible to handle the problem analytically. Moreover: the first property makes the boundary conditions (finite size sample) a crucial ingredient of the problem (i.e. the dipolar energy is not only a property of the material but also of the object shape); and beyond the simple case of a single pair of moments, the second property makes the prediction of the actual kind of ordering not intuitive, as it is strongly dependent on the locations of the moments on a lattice.

In this model the energy of the system is a function of the unit vectors $\boldsymbol{e}_{i}$ (pointing in the direction of the $i$-th dipole). At zero temperature the equilibrium state of the system is the state with $H$ being at minimum. At equilibrium the torque acting on any dipole,

$$
\boldsymbol{\tau}_{i}=\boldsymbol{e}_{i} \times\left\{\sum_{j=1 ; j \neq i}^{N} \frac{1}{R_{i j}^{3}}\left[3\left(\boldsymbol{e}_{j} \cdot \boldsymbol{e}_{i j}\right) \boldsymbol{e}_{i j}-\boldsymbol{e}_{j}\right]+\boldsymbol{b}\right\}
$$

must be equal to zero. To achieve this state any dipole step by step is turned by a small angle proportional to the acting torque $\mathrm{d} \boldsymbol{\alpha}_{i} \propto \boldsymbol{\tau}_{i}$ : one static relaxation step consists of turning any dipole of the system parallel to the torque acting on it. In this sense the SR method is similar to the MC method - the only difference is that the SR attempt is successful while in MC method the new configuration is adopted with a certain probability.

\section{Results and discussion}

\subsection{The ground state of the system at zero magnetic fields}

In order to better understand the self-behavior of a magnetic dipole system in the present study we basically consider the system in the absence of an external magnetic field. First of all we consider a linear model (a one-dimensional lattice) at zero temperature. Its ground state energy $\left(\boldsymbol{e}_{i}=\boldsymbol{e}_{x}, i=1,2, \ldots, N\right)$ can be easily calculated analytically

$$
H=-2 N \sum_{j=1}^{\infty} \frac{1}{j^{3}}=-2 N \zeta(3)=-2.404 N .
$$

Here $\zeta(3)=\sum_{j=1}^{\infty} \frac{1}{j^{3}}=1.202$ is the value of the zeta function for its argument being equal to 3 , thus the energy per dipole is $-2.404 E_{0}$. Almost the same energy $(-2.400$ per dipole) is found by the SR method. Analytical calculation of the ground state of the two- or three-dimensional model is a complicated problem, however it is easily done by the SR method.

In the present study for the sake of simplicity we consider dipole models forming an equidistant simple line lattice (one-dimensional model), a simple rectangle lattice (two-dimensional model) or a simple cubic (sc) lattice (three-dimensional model), with lattice parameters equal to 1. Calculations show that the ground state of the two-dimensional model is a system of parallel dipole lines being at the "ground state" in the one-dimensional model; however the dipoles in the neighbor lines are in opposite directions. In this model the calculated energy is -2.550 per dipole. Indeed, this value depends on the size of the model, the interaction range of dipoles and the applied boundary conditions: the presented value corresponds to a $100 \times 100$ dipole system with periodic boundary conditions. For the same model with a free boundary condition the calculated energy of the ground state due to the boundary effects is slightly lower, -2.5485 per dipole. The ground state of the sc three-dimensional model $(20 \times 20 \times 20$ dipoles $)$ is a set of parallel planes of the two-dimensional model being at their ground state; however, the neighbor planes are oriented antiparallel to each other. The calculated energy of the ground state of 
the three-dimensional model is -2.679 (periodic boundary conditions) and -2.600 per dipole for the free boundary conditions. These calculations show that the major part of the energy of a magnetic dipole system is formed by the linear chains of dipoles.

\subsection{Metastable states at zero temperature}

Numerical simulations by the SR method show that there are a number of metastable states which energetically are very close to the ground state. The reason for the existence of metastable states is that the ground state of a magnetic dipole chain is degenerated: the alignment of the dipoles in the chain to the left or to the right at the absence of magnetic field from any point of view is equivalent. Thus the energy of a dipole chain consisting of two or more segments with different dipole orientations is almost equal to the energy of a single line segment $(-2.404$ energy units per dipole). The segments are separated by a very thin "domain wall" consisting of a few dipoles the orientation of which gradually changes from one direction to the opposite one.

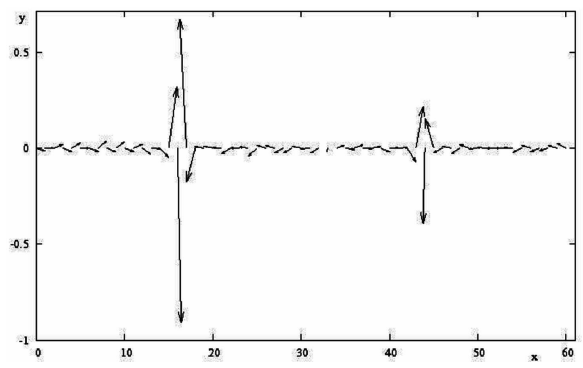

Fig. 1. Projection of the dipole moments (unit vectors) on the $x-y$ plane of a one-dimensional magnetic dipole model consisting OF 61 dipoles located at the sites of a simple one-dimensional lattice.

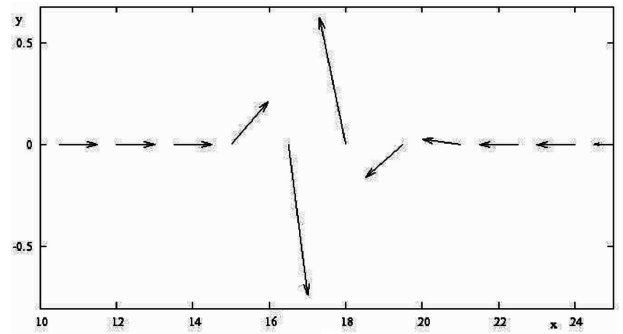

Fig. 2. Projection of the dipole moments on the $x-y$ plane of the first domain wall formed in the model shown in Fig. 1.

An example of a linear model at metastable state is shown in Fig. 1: it is a line of dipoles (along the $x$-axis) with dipole moments projected on the $x-y$ plane and consists of three segments oriented differently. The energy of this state is -2.334 per dipole. The magnetic structure of the metastable state is similar to the domain structure of a ferromagnetic material.

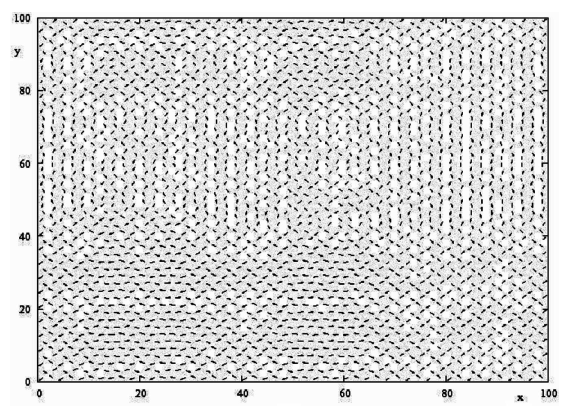

Fig. 3. Projection of the dipole moments on the $x-y$ plane of $50 \times 50$ dipoles model at zero temperature and in a zero magnetic field. Periodic boundary conditions are applied. The unit distance along the $x$ and $y$ axis is the double lattice parameter of the model.

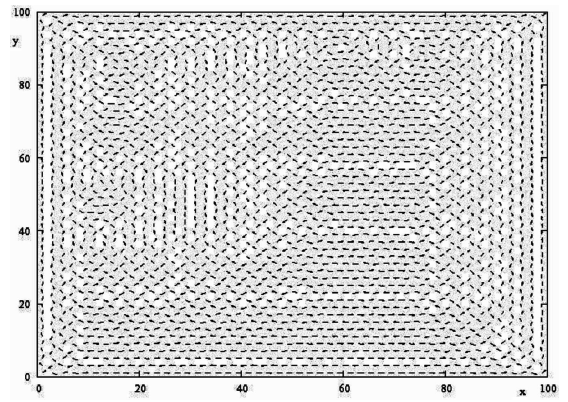

Fig. 4. Projection of the dipole moments on the $x-y$ plane of $50 \times 50$ dipoles model at zero temperature and in a zero magnetic field. Free boundary conditions are applied. The unit distance along the $x$ and $y$ axis is the double lattice parameter of the model.

Figure 2 shows such a one-dimensional domain. Its width turns out to be of the order of the dipole-dipole interaction length. The number of domains in the model depends on many factors such as the length of the model, starting configuration and boundary conditions. Therefore, depending on these conditions a number of

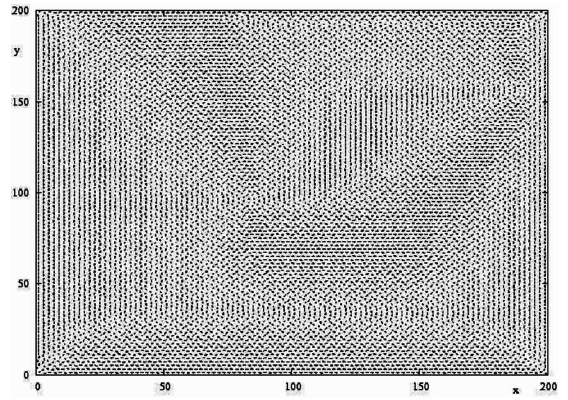

Fig. 5. Projection of the dipole moments on the $x-y$ plane of $100 \times 100$ dipoles model at zero temperature and in a zero magnetic field. Free boundary conditions are applied. The unit distance along the $x$ and $y$ axis is the double lattice parameter of the model. 


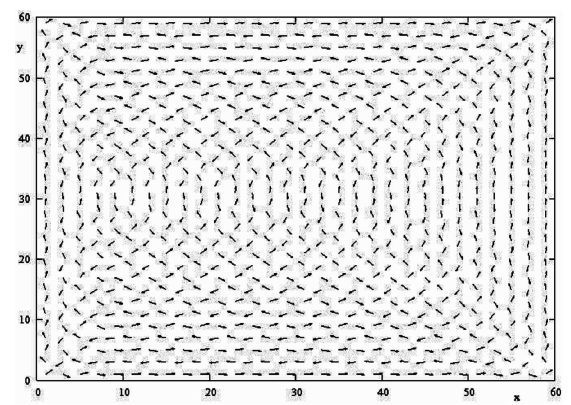

Fig. 6. Projection of the dipole moments on the $x-y$ plane of $30 \times 30$ dipoles model at zero temperature and in a zero magnetic field. Free boundary conditions are applied. The unit distance along the $x$ and $y$ axis is the double lattice parameter of the model.

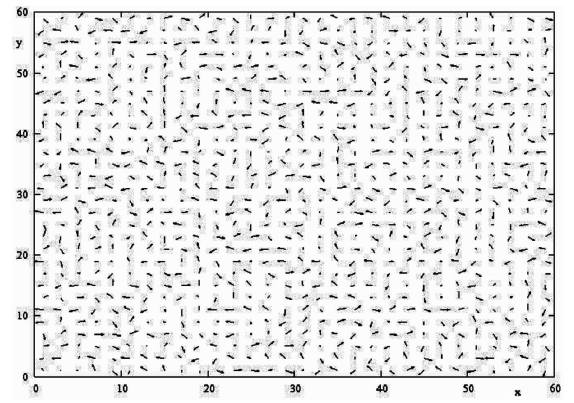

Fig. 7. Projection of the dipole moments on the $x-y$ plane of $30 \times 30$ dipoles model; reversed temperature $E_{0} / k_{\mathrm{B}} T$ is equal to 1.0 , magnetic field is absent. Free boundary conditions are applied. The unit distance along the $x$ and $y$ axis is the double lattice parameter of the model.

metastable states are rendered possible all energetically very close to each other. This in turn makes the theoretical investigation of the model difficult. From point of view of magnetic ordering the ground state of the single segment is perfectly ordered (all dipole moments are in the same direction and the system is with a maximum magnetization equal to 1 in dimensionless units). The metastable state at zero temperature is ordered too, however the correlation length now is limited. The lengths of the domains in the chain are on average equal to each other; therefore the average magnetization is zero. Thus the one-dimensional dipole model behaves like a classical superparamagnetic. However, depending on the "history" of the model, fluctuations of the size of the domains are possible and the model can be in such a metastable state when it behaves like a ferromagnetic.

\subsection{The two-dimensional model}

In order to illustrate the behavior of the magnetic dipole system more effectively, we present some graphical results for the two-dimensional model. Figures 3-5 $(50 \times 50$ and $100 \times 100$ dipoles, square lattice) demonstrate the influence of the boundary conditions and the size of

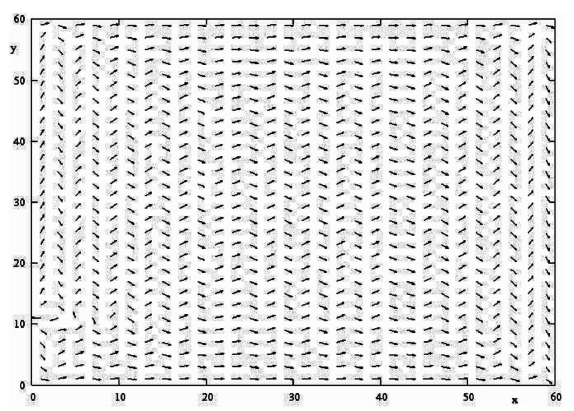

Fig. 8. Projection of the dipole moments on the $x-y$ plane of $30 \times 30$ dipoles model at zero temperature and with the magnitude of magnetic field $b=1$, applied along the $x$-axis. Free boundary conditions are applied. The unit distance along the $x$ and $y$ axis is the double lattice parameter of the model.

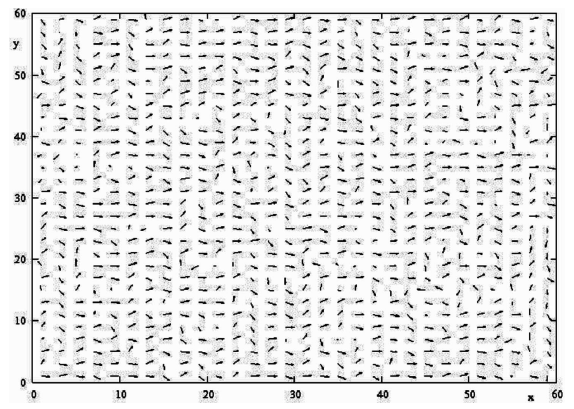

Fig. 9. Projection of the dipole moments on the $x-y$ plane of $30 \times 30$ dipoles model at reversed temperature $E_{0} / k_{\mathrm{B}} T=1.5$ and with the magnitude of magnetic field $b=1$, applied along the $x$-axis. Free boundary conditions are applied. The unit distance along the $x$ and $y$ axis is the double lattice parameter of the model.

the model on the magnetic structure of the model. Figures show the projections of the dipole moments (unit vectors) on the $x-y$ plane. According to FigS. 3-5 the magnetic structure of the system consists of antiferromagnetic domains with antiferromagnetic domain walls. The boundary conditions crucially influence the interior of the model, thus yielding different metastable states of the system, while the size of the model is less important.

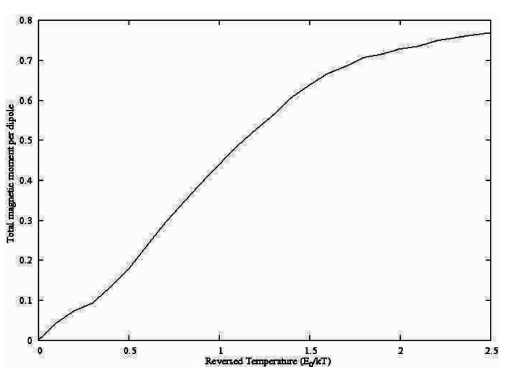

Fig. 10. Magnetization as a function of reversed temperature. 
In order to better see these effects, in Figs. 6, 7 we present a smaller model $(30 \times 30$ dipoles $)$. The state presented in Fig. 7 is obtained by MC simulation; the reverse temperature is in $E_{0} / k_{\mathrm{B}} T$ units. At non-zero temperature the magnetic structure consists of short line chains of parallel dipole forming some kind of antiferromagnetic aggregations. Figures 8, 9 demonstrate the behavior of the $30 \times 30$ dipole model in the presence of an external magnetic field and the influence of temperature. In the shown examples the magnetic field is along the $x$ -direction and its magnitude $b=1$. For completeness in Fig. 10 we present the magnetization of the above $30 \times 30$ dipole model as a function of the reverse temperature: the figure shows definitely the presence of some kind of order-disorder transition in the system in the vicinity of $E_{0} / k_{\mathrm{B}} T=1.2$; however, for the limited size of the system this transition is quite smooth.

\section{Conclusion}

The ground state of a dipole magnetic system forming a simple cubic (or square) lattice is a set of parallel dipole lines where the magnetic dipole at neighbor line are in opposite directions. In practice however the system at zero temperature is in a metastable state which consists of domains formed by antiparallel line segments.

\section{Acknowledgments}

This work was supported by the Ministry of Industry and Trade of Republic of Turkey (SanTez project N 00185.STZ.2007-2).

\section{References}

[1] M.F. Hansen, S. Morup, J. Magn. Magn. Mater. 184, 262 (1998).

[2] J.L. Dormann, L. Bessais, D. Fiorani, J. Phys. C 21, 2015 (1988).

[3] S. Morup, E. Tronc, Phys. Rev. Lett. 72, 3278 (1994).

[4] J.L. Dormann, D. Fiorani, E. Tronc, Adv. Chem. Phys. 98, 283 (1997).

[5] J.L. Dormann, D. Fiorani, E. Tronc, J. Magn. Magn. Mater. 202, 251 (1999).

[6] F.C. Fonseca, G.F. Gya, R.F. Jardim, R. Muccillo, N.L.V. Carreno, E. Longo, E.R. Leite, Phys. Rev. B 66, 104406 (2002).

[7] A. Fnidiki, C. Dorien, F. Richomme, J. Teillet, D. Lemarchand, N.H. Duc, J. Ben Youssef, H.Le. Gall, J. Magn. Magn. Mater. 262, 368 (2003).

[8] I.T. Iakubov, A.N. Lagarkov, S.A. Maklakov, A.V. Osipov, K.N. Rozanov, I.A. Ryzhikov, V.V. Samsonova, A.O. Sboychakov, J. Magn. Magn. Mater. 321, 726 (2009).

[9] V.V. Samsonova, A.O. Sboychakov, J. Magn. Magn. Mater. 321, 2707 (2009). 